\title{
Gestão da inovação em bibliotecas: elementos fundamentais de revisão de literatura internacional
}

\author{
Murilo Mauro Silveira* \\ William B. Vianna* \\ Sandra Rolim Ensslin
}

Artículo recibido:

13 de octubre de 2016

Artículo aceptado:

28 de octubre de 2016
Resumo

O objetivo desse estudo é identificar e analisar aspectos relevantes da literatura internacional sobre gestão da inovação em Bibliotecas. Justifica-se pela necessidade de avanços e melhorias na gestão da informação em Bibliotecas para atender novas demandas sociais. Trata-se de um estudo exploratório, de matriz qualitativa e fundamento construtivista que se valeu da metodologia ProKnow-C (Knowledge Development Process - Constructivist) para seleção, identificação/análise da literatura. Resultou na identificação de 14 artigos, diversos periódicos e elementos de tesauro relevantes e fundamentais para evolução do conhecimento sobre inovação em Bibliotecas.

* Instituto Federal de Santa Catarina, Brasil.

willscut2@hotmail.com

** Universidad Federal de Santa Catarina, Brasil.

william.vuanna@ufsc.br sensslin@gmail.com.br

INVESTIGACIÓN BIBLIOTECOLÓGICA, vol. 32, núm. 76, julio/septiembre, 2018, México, ISSN: 2448-8321 pp. 29-44 
Palavras-chave: Gestão da Inovação em bibliotecas; Proknow-C; Gestão da Informação.

\section{RESUMEN}

El objetivo de este estudio es identificar y analizar los aspectos relevantes de la literatura internacional sobre gestión de la innovación en las bibliotecas. Se justifica por la necesidad de avances y mejoras en la gestión de la información en las bibliotecas para satisfacer las nuevas demandas sociales. Se trata de un estudio exploratorio de la matriz cualitativa y fundamento constructivista que hizo uso de la metodología ProKnow-C (Proceso de Desarrollo del Conocimiento-constructivista) para la selección, identificación/ análisis de la literatura. El resultado fue la identificación de 14 artículos, varias revistas y elementos de sinónimos relevantes y fundamentales para el avance del conocimiento en la innovación en las Bibliotecas.

Palabras clave: Gestión de la Innovación en Bibliotecas; Proknow-C; Gestión de la Información.

Innovation management in libraries: fundamental elements of reviewing the international literature Murilo Mauro-Silveira, William B.-Vianna and Sandra Rolim-Ensslin

\section{Abstract}

The aim of this study is to identify and analyze relevant aspects of international literature on innovation in Libraries. It justified by the need for advances and improvements in information management in libraries to meet new social demands. It is an exploratory study of qualitative matrix and constructivist foundation that made use of ProKnow-C methodology (Knowledge Development Process - Constructivist) for selection of literature, identification/analysis of the literature. It resulted in the identification of 14 articles, several journals and relevant thesaurus elements and critical to advancement of knowledge on innovation in Libraries.

Keywords: Innovation Management in Libraries; 


\section{INTRODUÇÃO}

Crente aos atuais desafios da globalização e da competitividade, a ino1 vação tem se tornado foco de diversas organizações seja de pequeno, médio ou grande porte; constituindo-se, assim, um relevante fator de sucesso (Gonçalves et al., 2013). E a inovação só será bem sucedida se os usuários finais ficarem satisfeitos, e para isso as organizações necessitam se orientar pelo atendimento das demandas dos mesmos (Georgy, 2010a).

Nesse contexto, as bibliotecas também enfrentam novas pressões econômicas e sociais para que os serviços de informação sejam capazes satisfazer constantemente as necessidades de informações reais (explicitas ou implícitas), bem como atendam as expectativas dos usuários (Kostagiolas et al., 2011).

De acordo com Georgy (2010a), as bibliotecas podem se posicionar com sucesso no futuro pela adesão a uma gestão moderna, baseada e adaptada para suas particularidades e fundamentada na gestão da inovação. Entretanto, Rowley (2011) alerta que, embora as bibliotecas precisem inovar, há pouca evidência de discussão sobre a gestão e seus processos de inovação na literatura em Biblioteconomia.

Para Leonard e Clementson (2012), o que está claro a partir da investigação que tem sido feita até agora em matéria de inovação em bibliotecas, é que pouco sabemos sobre este tema, e que os resultados não só são variados, como não possuem um corpo substancial de pesquisas empíricas, sendo difícil construir uma teoria.

Diante dessa problemática, pergunta-se: que informações científicas sobre a gestão da inovação em bibliotecas podem subsidiar o desenvolvimento do tema em Ciência da Informação e Biblioteconomia?

O objetivo do estudo foi identificar e analisar fragmentos relevantes da literatura internacional sobre a gestão da inovação em bibliotecas a partir de bases internacionais indexadas para expansão do conhecimento no campo da Ciência da Informação e Biblioteconomia.

A justificativa fundamenta-se na afirmação de Leonard e Clementson (2012), para quem há poucas pesquisas ligadas diretamente a estrutura organizacional que apoia a inovação ou as características inovadoras das pessoas que trabalham em bibliotecas, embora a inovação pareça ser de interesse geral e crescente.

Trata-se de um estudo exploratório, de natureza predominantemente qualitativa, fundamentado no construtivismo. A pesquisa exploratória auxilia quando o tema escolhido é pouco explorado e torna-se difícil sobre ele 
formular hipóteses precisas e operacionalizáveis e essa pode ser a primeira etapa de um projeto maior (Gil, 1999).

Segundo Sampieri, Collado e Lucio (1994), o uso do estudo exploratório é útil quando o objetivo é examinar um determinado tema que tenha sido pouco ou nada estudado anteriormente, para obter um maior grau de familiaridade com os fenômenos estudados. Caracteriza-se ainda como estudo qualitativo, pois, de acordo com Creswell (2014), a visão de mundo do pesquisador é que dá sentido às interpretações em que o mundo é representado.

E construtivista porque como afirma Roy (1993), essa perspectiva contribui com os participantes por meio de um conjunto de chaves, com base em objetivos e valores, identificando sistemas que ajudam a compreender contextos em que eles pretendem intervir.

\section{O instrumento de intervenção: Knowledge Development Process- Constructivist (ProKnow-C)}

Para seleção, orientação, identificação, análise e reflexão sobre as características da literatura foi utilizado o instrumento de intervenção Knowledge Development Process-Constructivist (ProKnow-C). Trata-se de um processo para busca e análise da literatura desenvolvido no Laboratório de Metodologia Multicritério em Apoio à Decisão (LabMCDA), da Universidade Federal de Santa Catarina (UFSC), Brasil; e objetiva construir conhecimento sobre um tema a partir dos interesses e delimitações do pesquisador no uso de filtros para identificação de um portfólio bibliográfico $(\mathrm{PB})$ relevante conforme demonstra a figura a seguir (Ensslin et al., 2010; Dutra et al., 2015; Ensslin et al., 2013):

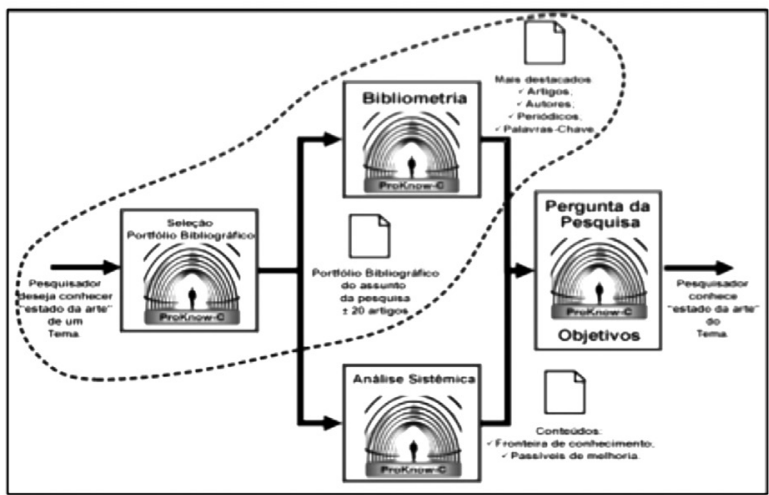

Figura 1. Etapas da metodologia aplicada na pesquisa 
O processo é composto por quatro etapas: 1) seleção de um portfólio de artigos sobre o tema da pesquisa em bases de dados relevantes; 2) análise de aspectos bibliométricos básicos do portfólio; 3) análise sistêmica e 4) definição da pergunta e objetivo de pesquisa (Ensslin et al., 2014).

Conforme delimitado na figura 1, esse estudo valeu-se das duas primeiras fases propostas pelo Proknow-C sobre a análise da literatura internacional sobre a gestão da inovação em bibliotecas disponíveis gratuitamente em acesso aberto no portal de periódicos da Coordenação de Aperfeiçoamento de Pessoal de Nível Superior (Capes), realizada em abril de 2015, no espaço temporal de dez anos, ou seja, de 2004 a 2014. O instrumento foi selecionado por sua ergonomia e simplificação dos processos para pesquisadores em geral.

Conforme a figura 1, a análise bibliométrica se limitou a aspectos primários, ou seja, teve como objetivo verificar as características básicas das publicações na área do conhecimento do que está sendo investigado, com o intuito de agregar conhecimento ao pesquisador para que este saiba onde e como buscar informações sobre o tema (Dutra et al., 2015). A partir dos aspectos primários foram identificadas contribuições qualitativas para o estudo do tema e possibilidades para o seu desenvolvimento nas etapas 3 e 4 da metodologia proposta.

Para aferir validade aos resultados de cada uma das etapas, fez-se uso das estratégias "membro de verificação" e "auditor externo". No caso, um dos autores mais experiente no processo e de outra área de conhecimento, participou da pesquisa e procedeu uma auditoria no desenvolvimento de cada atividade para validar os procedimentos realizados, bem como aferir se os resultados das etapas desenvolvidas pelos envolvidos na mesma investigação separadamente, convergiam; como, por exemplo, as mesmas palavras-chave para representar os eixos de um tópico e favorecer a replicabilidade (Creswell, 2010; 2014; Gibbs, 2007).

\section{A GESTÃO DA INOVAÇÃO E SUA APLICAÇÃO A BIBLIOTECAS}

Para Tidd et al. (2008), a inovação é o resultado de um conjunto de processos, cujos elementos favorecem a ocorrência de avanços científicos e tecnológicos, cujos elementos centrais são baseados em conhecimento, informação e criatividade:

a inovação, de fato, não é algo gerenciável em si. Trata-se de um resultado e, como tal, decorre de um processo. O processo, por sua vez, é gerenciável e, portanto, passível de acompanhamento e controle em suas etapas. Embora a inovação 
possa conter conhecimento, informação e criatividade, é no processo de inovação que tais elementos afloram e podem ser planejados, acompanhados e gerenciados (Dallagnol, 2010).

Para Munro et al. (2011), os processos de inovação a serem gerenciados envolvem produtos, processo e estratégia sendo que: 1) a inovação de produtos é o processo de desenvolvimento de novos produtos ou serviços que melhoram a experiência do usuário; 2) a inovação de processos aborda a medição e estruturação de atividades institucionais para alcançar melhorias no desempenho dos serviços; 3) a inovação estratégica retrata modelos desafiadores existentes de atingir o valor do cliente, a fim de atender a recém-emergentes necessidades dos clientes.

Há ainda que se distinguir entre inovações incrementais e radicais. A inovação radical é uma mudança fundamental, realizada por meio de um projeto específico e tipicamente associada com o desenvolvimento de um novo produto ou serviço. Uma inovação incremental é uma adição para uma inovação anterior sem mudar o seu conceito essencial, uma melhoria ou aperfeiçoamento de algo existente (Rowley, 2011).

Outros elementos que permitem o gerenciamento da inovação são as ferramentas que a apoiam. O octógono da inovação é uma proposta de ferramenta para diagnóstico do potencial inovador, bem como para a gestão de empresas inovadoras e é composto pelos elementos: estratégia, liderança, funding, processo, estrutura, pessoas, cultura, relacionamentos (Scherer e Carlomagno, 2009).

Para Georgy (2010a), o aumento da concorrência e da dinamicidade da Ciência da Informação e dos serviços de bibliotecas também forçam as bibliotecas permanentemente a optar para desenvolvimento de soluções negociáveis, novas e otimizadas aos clientes, isto é, a busca por inovação.

De acordo com Jianzhong e Chen (2013), a boa biblioteca, nos velhos tempos dourados, era uma biblioteca de dezenas de milhares de itens de livros, onde havia espaço de leitura suficiente para as pessoas a encontrar e ler livros confortavelmente, e era capaz de atender a demanda dos usuários. Mas hoje, a biblioteca tem de ser agressiva e dinâmica. Esta dinamicidade deve-se a diversos fatores pois a biblioteca está num período de transição para uma idade eletrônico-e-globalizado. Conforme Kostagiolas et al. (2011), hoje em dia, as persistentes mudanças no ambiente econômico, junto com o advento das tecnologias, aponta para necessidade de implementar novos modelos de gestão que incorporem as necessidades dos usuários como prioridade na filosofia dos serviços de biblioteca. 
Para Deiss (2004) as barreiras à inovação em organizações mais maduras ou envelhecidas são maiores, pois tem mais dificuldades em correr risco, experimentar e criar. E criar valor em serviços oferecidos ao seu público é a principal razão para inovar em uma instituição sem fins lucrativos para a publico o valorize. Para o autor, as bibliotecas por serem instituições na maioria sem fins lucrativos entregam a comunidade e aos usuários o valor social. Estes fatores tornam a liderança e desenvolvimento para inovação, tanto importantes como difíceis.

A formulação de uma gestão proposta a mudar a realidade em que as bibliotecas situam-se precisa ser capaz de alterar alguns paradigmas, sendo que os gestores das bibliotecas abordaram sobre como é necessária a inovação para atender a evolução das necessidades dos clientes. As razões para inovar podem ser vistas como uma escolha entre as práticas tradicionais e a re-imaginação dessas práticas para melhor responder as novas circunstâncias em um ambiente de mudanças, apesar de barreiras como estrutura hierárquica tradicional presente em bibliotecas que pode dificultar a cultura empreendedora (Leonard e Clementson, 2012).

Uma das mensagens importantes é que as bibliotecas precisam olhar para além da gestão operacional e ter uma carteira de inovação que contemple uma cultura de inovação, desenvolvimento de lideranças e equipes criativas, relações de colaboração e processos para capitalizar o envolvimento dos usuários. inovação pode consumir recursos consideráveis, envolver muitas pessoas, e tem potencialmente consequências significativas para uma biblioteca, assim a inovação não pode simplesmente ser deixada ao acaso, ela necessita ser gerenciada (Rowley, 2011).

Frente a uma reconhecida dinâmica de mudanças, a necessidade e importância de inovações nas organizações e diante de algumas barreiras, abordar a inovação em bibliotecas pode ser compreendido mais como uma necessidade do que uma novidade. É nesse sentido que identificar aspectos bibliométricos básicos, bem como sistematizar um fragmento da literatura sobre o assunto pode trazer contribuições significativas para o desenvolvimento do tema, conforme se apresenta a seguir.

\section{RESUlTADOS QUANTITATIVOS EXTRAÍDOS DO PB E SUAS REFERÊNCIAS}

Esta seção apresenta o mapeamento da literatura sobre gestão da inovação e bibliotecas a partir do Portfólio Bibliográfico (PB) selecionado conforme prevê o Proknow-C e inicia-se com a seleção do banco de artigos brutos, passando por uma filtragem nos artigos recuperados, para posterior etapa, o teste 
de representatividade. Os resultados gerais do processo encontram-se na figura a seguir:

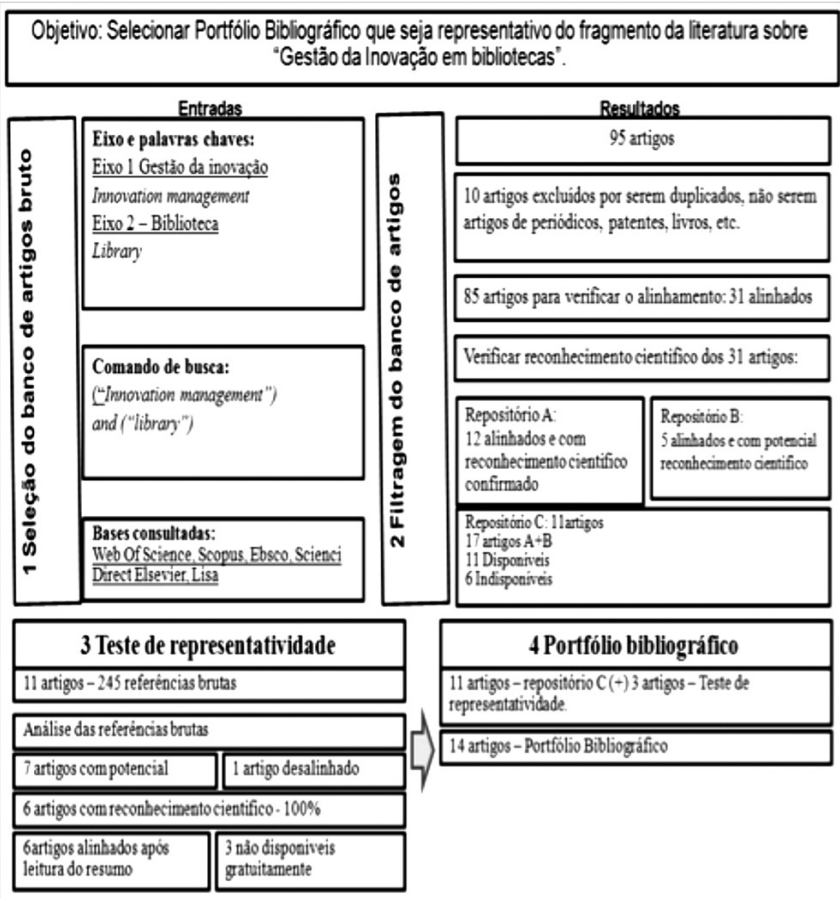

Figura 2. Processo de seleção do PB Fonte: elaborado pelo autores (2015)

A busca nas bases internacionais apresentadas na figura 1 (WoS, Scopus, EBsco, Science Direct, Elsevier e Lisa), resultou em noventa e cinco (95) artigos brutos, sendo dez (10) excluídos por não serem artigos e trinta e um (31) foram considerados inicialmente alinhados, mas catorze (14) foram descartados após leitura do título e/ou aferição do não reconhecimento científico, evidenciada pela quantidade de citações de cada artigo (menos de duas citações) em outros trabalhos científicos.

Dos doze (12) artigos restantes, um estava duplicado, sendo que fez-se um teste de representatividade que consistiu na análise das referências contidas nos onze artigos (11) primários, onde foram identificadas duzentas e quarenta e cinco (245) referências brutas a partir das quais identificou-se-: 1) avaliação quanto ao alinhamento do título do artigo ao tema da pesquisa; 2) enquadramento ao mesmo espaço temporal utilizado para seleção do $\mathrm{PB}$; 3 ) retirada de referências duplicadas e que não são artigos de periódicos científicos. 
Pela análise das referências brutas constatou-se que sete (7) artigos tinham potencial para incorporar o banco de artigos primários do portfólio bibliográfico e uma não se tratava de artigo de periódico e foi excluída.

O passo seguinte foi do teste de representatividade que identificou o número de citações desses seis (6) artigos, os quais foram ordenados por ordem decrescente da quantidade de citações gerais e consistiu no levantamento de todas as referências bibliográficas dos artigos, restringindo-se ao espaço temporal considerado para o estudo (2004-2014) e a artigos publicados em periódicos, a partir do que fez-se uma nova consulta ao sítio Google Scholar para identificar a quantidade de citações feitas aos artigos das referências do PB.

Após essa fase, reorganizou-se o resultado, classificando o conteúdo por número de citações de forma decrescente a partir do grau de representatividade em percentuais de cada artigo em relação ao total de referências, ou seja, 100\% das citações totais a fim de identificar se os artigos do PB continham essas referências.

Foram destacados seis (6) artigos para a leitura completa que três (3) deles não estavam disponíveis gratuitamente e que nenhum dos demais textos estavam desalinhados, após sua leitura integral. Assim, o portfólio bibliográfico foi formado com três (3) artigos mais onze (11) já contidos no repositório primário, ou seja, catorze artigos (14) relevantes para busca. Os artigos foram analisados com o propósito de quantificar e identificar características básicas relevantes do PB (14 artigos) e de suas referências (245 artigos) que são apresentados em gráficos a seguir a partir da autoria, seguida de periódicos relevantes e outros elementos:

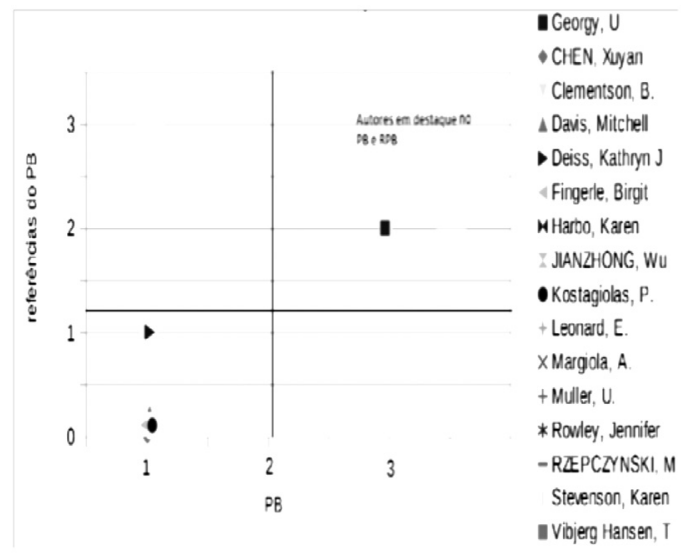

Gráfico 1. Autores em destaque no PB e nas referências do PB (RPB) Fonte: elaborado pelo autores (2015) 
No que se refere a autoria, dentre os dezesseis (16) autores do PB e referências destacou-se com três publicações: Ursula Georgy (2012), seguida de Kathryn J. Deiss (2004), que obteve o segundo lugar. A autora destacada primeiro é Dra. em química, lecionou na Fachhochschule Köln, na Universidade de Ciências Aplicadas de Niederrhein e desde 2000, dá aulas na Universidade de Ciências Aplicadas de Colônia na Alemanha, do Instituto de Informação em Ciências da Informação e Marketing. A partir de setembro de 2011 dirigiu o Centro de Educação e a Biblioteca de Ciência da Informação do Instituto de Ciência da Informação da FH Köln (Georgy, 2012; Deiss, 2004).

Os interesses de pesquisa da autora são: a gestão de serviços de informação, a fidelização de clientes, o perfil e marca de serviços de informação, a fixação de preços dos serviços de informação, análise estratégica de patentes e gestão da inovação, especialmente a inovação aberta e qualidade. A seguir é possível visualizar os dados sobre periódicos.

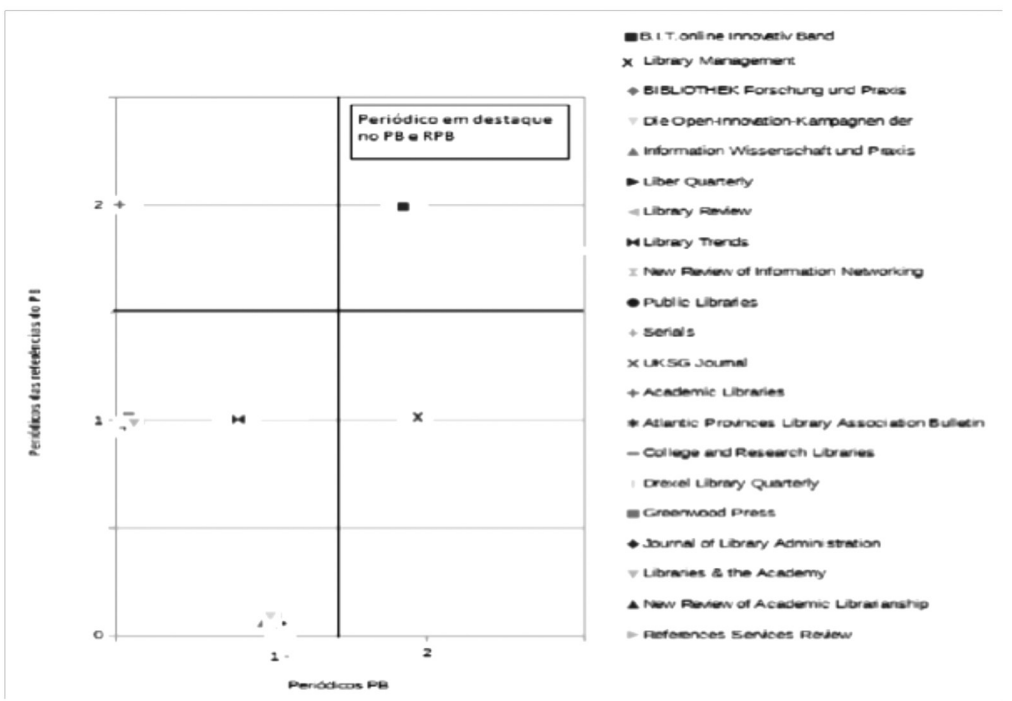

Gráfico 2. Análise dos periódicos em destaque no PB e referência do PB (RPB)

Fonte: elaborado pelo autores (2015)

Dentre os 14 artigos que compreendem o $\mathrm{PB}$, os periódicos que tiveram maior destaque foram el: Library Management e B.I.T. Online Innovativ Band com duas indicações cada. O jornal Library Management (LM) publica artigos de interesse dos gestores de biblioteca e acadêmicos e possui mais de onze mil downloads por mês. $L M$ reflete as mais recentes pesquisas realizadas em 
meio acadêmico, governo e instituições corporativas com relatórios de pensamento contemporâneo e implicações práticas.

O periódico trata também de gestão estratégica, o uso da informação, qualidade e gestão da mudança, as questões de gestão e marketing, automação, finanças de biblioteca e medição do desempenho.

O segundo periódico mais relevante, o jornal B.I.T. Online Innovativ Band, com presença atual na internet: <www.bit-online.de $>$ é um jornal Alemão sobre bibliotecas e aborda temas atuais de desenvolvimentos, bem como em aplicações técnicas em biblioteconomia. Foi fundado em 1998 e é publicado trimestralmente em Wiesbaden Verlag por Dinges \& Frick.

Surgiu a partir do crescimento e uso emergente da internet e possui contribuições de especialistas na área de biblioteconomia, bibliotecas, a ciência de arquivo e a utilização de modernos sistemas de informação e fomenta um prêmio de inovação em bibliotecas. Outro ponto de relevância analisado são as palavras chaves mais citadas no $\mathrm{PB}$ conforme o gráfico a seguir:

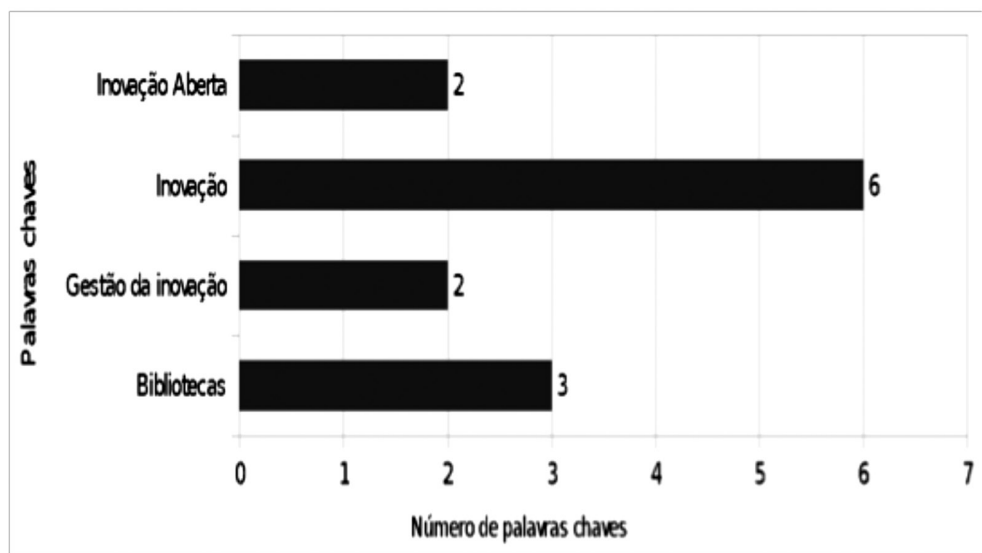

Gráfico 3. Palavras chaves do PB

Fonte: elaborado pelo autores (2015)

$\mathrm{Na}$ identificação das 43 palavras-chave, a que mais se destacou foi 'inovação', com 6 ocorrências, seguida de bibliotecas com 3 ocorrências. As demais com 2 ocorrências foram: gestão da inovação e inovação aberta. A seguir os resultados por tipo de biblioteca: 


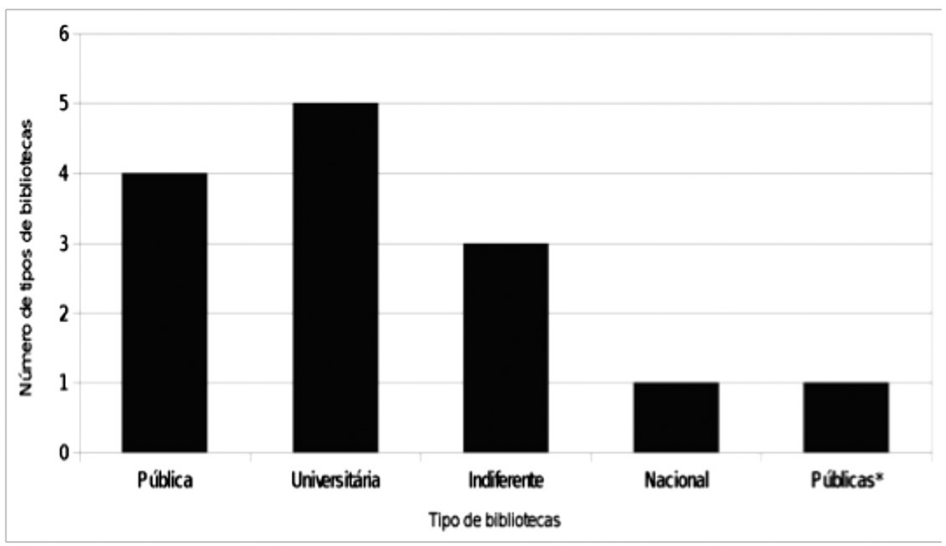

Gráfico 4. Tipos de bibliotecas Fonte: elaborada pelo autores (2015)

Os tipos de biblioteca de acordo com sua finalidade são em geral: nacionais, públicas, universitárias, especializadas, escolares, especiais, dentre outras. Mas nesse estudo os resultados apontaram predominantemente para três tipos de bibliotecas: nacionais, públicas e universitárias (Oliveira e Valadares, 2005).

Destaca-se que as pesquisas se concentram em bibliotecas universitárias e públicas. Nenhum artigo salientou como local de abordagem bibliotecas especializadas, escolares, infantis, $\mathrm{d}$ entre outras. Vale destacar que bibliotecas "Públicas", no caso, referiam-se a bibliotecas que possuem como órgão gestor o poder público, não necessariamente quanto ao acesso.

Resultados QUALitativos: CONTRibuiçÕes E DESAFIOS FUtUROS PARA o desenvolvimento do tema na Ciência da Informação e Biblioteconomia

Para subsidiar o desenvolvimento do tema na Ciência da Informação e na Biblioteconomia, ao menos dois resultados qualitativos são relevantes e emergem da análise realizada. Primeiramente a respeito da importância em considerar as variáveis específica de cada tipo de biblioteca em cada contexto e o segundo é o próprio corpo teórico e sua fundamentação expressos nos catorze (14) artigos selecionados. Apresenta-se, a seguir um quadro categorizado de acordo com Georgy (2010a), para quem o processo de inovação envolve fatores externos e internos: 


\begin{tabular}{|l|l|l|}
\hline \multicolumn{1}{|c|}{ Fatores } & \multicolumn{1}{|c|}{ Autor } & \multicolumn{1}{c|}{ Descrição } \\
\hline \multirow{4}{*}{ Externos } & Harbo e Hansen (2012) & $\begin{array}{l}\text { Gestão focada nas necessidades } \\
\text { dos usuários. }\end{array}$ \\
\cline { 2 - 3 } & Fingerle e Firgerle (2012) & $\begin{array}{l}\text { Inovação aberta - usuários no } \\
\text { processo de inovação. }\end{array}$ \\
\cline { 2 - 3 } & Munro et al. (2011) & Tecnologia móvel - dispositivos. \\
\cline { 2 - 3 } & Georgy (2010a) & $\begin{array}{l}\text { Integração da qualidade e inovação } \\
\text { voltada para o cliente. }\end{array}$ \\
\hline \multirow{5}{*}{ Internos } & Davis (2013) & Mídias. \\
\cline { 2 - 3 } & Kostagiolas et al. (2011) & Modelo de resposta a gestão. \\
\cline { 2 - 3 } & Rzepczynski (2013) & $\begin{array}{l}\text { Inovação sobre o ponto de vista dos } \\
\text { diretores. }\end{array}$ \\
\cline { 2 - 3 } & Georgy (2010b) & Capacidade de inovar. \\
\cline { 2 - 3 } & Rowley (2011) & Abordagem estratégica. \\
\cline { 2 - 3 } & Jianzhong e Chen (2013) & Mudança de paradigmas. \\
\cline { 2 - 3 } & Deiss (2004) & Pensamento estratégico. \\
\hline
\end{tabular}

Quadro 1. Fatores externos e internos na gestão do inovação em bibliotecas Fonte: elaborado pelo autores (2015)

Cabe ressaltar que existem outras características ou mesmo formas de categorizar os resultados, inclusive propostas por autores do portfólio bibliográfico, a serem considerados e lacunas nesse sentido poderão ser abordadas com novas pesquisas ou reorganização, aprofundamento e expansão desse estudo. Por fim, considera-se relevante o próprio corpo teórico identificado nos catorze artigos do portfólio bibliográfico, apresentados a seguir:

\begin{tabular}{|c|l|}
\hline Artigo & \multicolumn{1}{|c|}{ Descrição } \\
\hline $\begin{array}{c}\text { Müller } \\
(2001)\end{array}$ & $\begin{array}{l}\text { Apresenta uma ferramenta de gestão, que pode ser utilizada para pesquisa } \\
\text { de mercado, gestão da inovação e satisfação de clientes. }\end{array}$ \\
\hline $\begin{array}{c}\text { Deiss } \\
(2004)\end{array}$ & $\begin{array}{l}\text { Explora a natureza da inovação, em especial no setor público, olhando para } \\
\text { o papel que o pensamento estratégico pode desempenhar na inovação. } \\
\text { Particularmente organizações sem fins lucrativos. }\end{array}$ \\
\hline $\begin{array}{c}\text { Georg } \\
(2010 a)\end{array}$ & $\begin{array}{l}\text { Descreve a interação de inovação e de gestão da qualidade orientada para o } \\
\text { cliente em bibliotecas. }\end{array}$ \\
\hline $\begin{array}{c}\text { Georgy } \\
(2010 b)\end{array}$ & $\begin{array}{l}\text { Avalia a capacidade inovadora e capacidade de inovação das bibliotecas } \\
\text { Alemãs e fornecedores de informações públicas; }\end{array}$ \\
\hline Kostagiolas etal. & $\begin{array}{l}\text { Descreve uma análise qualitativa para identificar o potencial de um modelo } \\
\text { (2011) }\end{array}$ \\
$\begin{array}{l}\text { de gestão como resposta a um caminho para a sobrevivência de bibliotecas } \\
\text { dentro da crise econômica da Grécia. }\end{array}$ \\
\hline
\end{tabular}




\begin{tabular}{|c|l|}
\hline $\begin{array}{c}\text { Munro et al. } \\
(2011)\end{array}$ & $\begin{array}{l}\text { Discorre sobre o uso de dispositivos móveis em bibliotecas, como dispositi- } \\
\text { vos de informação-chave para os usuários. }\end{array}$ \\
\hline $\begin{array}{c}\text { Rowley } \\
(2011)\end{array}$ & $\begin{array}{l}\text { Propõe um modelo holístico e uma abordagem estratégica com sete facetas } \\
\text { de uma estratégia de inovação. }\end{array}$ \\
\hline $\begin{array}{c}\text { Fingerle e Firgerle } \\
(2012)\end{array}$ & $\begin{array}{l}\text { Demonstra como os clientes podem ser integrados e participar em projetos } \\
\text { de inovação em bibliotecas. }\end{array}$ \\
\hline $\begin{array}{c}\text { Georgy } \\
(2012)\end{array}$ & $\begin{array}{l}\text { Aborda a Gestão Estratégica da Inovação em serviços dentro de biblioteca } \\
\text { e nas áreas de Ciência da Informação, independente do tamanho das } \\
\text { instituições (pequenas, médias, grandes), privadas, públicas. }\end{array}$ \\
\hline $\begin{array}{c}\text { Harbo e Hansen } \\
(2012)\end{array}$ & $\begin{array}{l}\text { Retrata como facilitar uma cultura de inovação dentro de bibliotecas acadê- } \\
\text { micas, com foco nas necessidades dos usuários. }\end{array}$ \\
\hline $\begin{array}{c}\text { Leonard e Clementson } \\
(2012)\end{array}$ & $\begin{array}{l}\text { Identifica as características inovadoras de bibliotecários de negócios e da } \\
\text { cultura organizacional de suas bibliotecas. }\end{array}$ \\
\hline $\begin{array}{c}\text { Davis } \\
\text { (2013) }\end{array}$ & $\begin{array}{l}\text { Identifica experiências de mídias em relação as bibliotecas. Como as } \\
\text { bibliotecas podem se comportar com relação aos mercados e alcançar seu } \\
\text { lugar nele. }\end{array}$ \\
\hline $\begin{array}{c}\text { Rzepczynski } \\
(2013)\end{array}$ & $\begin{array}{l}\text { Revisa e examina as necessidades e potencialidades para transição de uma } \\
\text { biblioteca de paradigma baseado em papel para todos os paradigmas de } \\
\text { mídia na nova era de mudanças. } \\
\text { (2013) }\end{array}$ \\
\hline diretores de bibliotecas públicas dos Estados Unidos da América. \\
\hline
\end{tabular}

Quadro 2. Fatores externos e internos na gestão do inovação em bibliotecas Fonte: elaborado pelo autores (2015)

Por fim, considera-se que o tema, apesar de emergente, revelou um escasso corpo teórico, haja vista os resultados quantitativos das citações, autores, periódicos e outros, o que aponta para possibilidade de maior desenvolvimento do tema na área. Para promover avanços e ampliação do corpo teórico identificado nesse estudo, sugere-se novas investigações que considerem outras perspectivas e palavras-chave relacionadas a: 1) aspectos institucionais como o tamanho, recursos financeiros, segmentos de atuação, aspectos geográficos; 2) aspectos internos como clima organizacional e cultura e perspectiva dos usuários; 3) avaliação da gestão da inovação e benchmarking.

E cabe ressaltar algumas limitações desse estudo: 1) restrito a artigos disponíveis gratuitamente à comunidade da UFSC, no portal Capes; 2) análise por palavras-chave pré-definidas pelos pesquisadores; 3) julgamento e interpretação dos autores a partir de seus interesses e objetivos; 4) universo e amostragem restrita para os filtros utilizados. 


\section{REFERÊNCIAS}

Creswell, J.W. 2014. Research design: Qualitative, quantitative, and mixed methods approaches, $4^{\mathrm{a}}$ ed. New York: Sage.

Creswell, J.W. 2010. Projeto de pesquisa: métodos qualitativos, quantitativos e mistos. Porto Alegre: Artmed.

Dallagnol, R.M. , 2010. "A gestão da inovação nas universidades: o capital social e a institucionalização de unidades de inovação no ambiente acadêmico”. Florianópolis: Departamento de Engenharia do Conhecimento, Programa de Pós-Graduação em Engenharia e Gestão do Conhecimento, Universidade Federal de Santa Catarina, tese de Doutorado em Engenharia e Gestão do Conhecimento.

Davis, M. 2013. "Doing well by doing good: How libraries can reclaim their role at the center of the information universe". Insights: The UKSG Journal 26 (2): 204-209.

Deiss, K.J. 2004. "Innovation and strategy: Risk and choice in shaping user-centered libraries". Library Trends 53: 17-32.

Dutra, A., V.M. Ripoll-Feliu, A.G. Fillol, S.R. Ensslin and L. Ensslin. 2015. "The construction of knowledge from the scientific literature about the theme seaport performance evaluation". International Journal of Productivity and Performance Management 64 (2): 243-269.

Ensslin, S.R., L. Ensslin, J.M. Imlau e L.C. Chaves. 2014. "Processo de mapeamento das publicações científicas de um tema: portfólio bibliográfico e análise bibliométrica sobre avaliação de desempenho de cooperativas de produção agropecuária”. Revista de Economia e Sociologia Rural 52: 587-608.

Ensslin, L., S.R.; Ensslin e H.M. Pinto. 2013. "Processo de investigação e análise bibliométrica: avaliação da qualidade dos serviços bancários”. Revista de Administração Contemporânea 17 (3): 325-349.

Ensslin, L., S.R. Ensslin, R.T.O. Lacerda and J.E. Tasca. 2010. "ProKnow-C, Knowledge Development Process Constructivist". Processo técnico com patente de registro pendente junto ao inpi. Brasil.

Fingerle, V.B. and B. Fingerle. 2012. "Innovation zum mitmachen: Die open innovation-kampagnen der zbw mit open innovation gemeinsam mit kundinnen und kunden neuerungen in bibliotheken fördern”. Bibliothek 36 (3).

Georgy, U. 2012. "Open innovation Integration der hochschulen in den innovations prozess von bibliotheken und information seinrichtungen". Information Wissenschaft und Praxis 63: 37-44.

Georgy, U. 2010a. "Qualität im service und dienstleistungs management von bibliotheken durch erfolgreiches innovations management". B.I.T. Online Innovativ, Band 30. Wiesbaden, Dinges and Frick: 33-55.

Georgy, U. 2010b. "Erfolg durch innovation". B.I.T.online Innovativ, Band 29. Wiesbaden, Dinges and Frick.

Gibbs, G. 2007. "Analysing qualitative data”. In U. Flick (ed.), Qualitative research kit. Londres: Sage.

Gil, Antonio Carlos. 1999. Métodos e técnicas de pesquisa social. $5^{a}$ ed. São Paulo: Atlas.

Gonçalves, L.C.; R.C. Moraes e V.G. Pereira. 2013. "Gestão da inovação em organizações um estudo bibliométrico”, em Congresso Internacional de Administração, 26. Ponta Grossa. Anais... Ponta Grossa: uepg, 2013. Disponível em <www.admpg.com.br/2013/down.php?id=145\&q=1>, acesso em 24 jun. 2015. 
Harbo, K. and T.V. Hansen. 2012. "Getting to know library users needs experimental ways to user-centred library innovation”. Liber Quarterly 21 (3-4): 367-385.

Jianzhong, W. and X. Chen. 2013. "Transition and transcendence: the innovative development of Shanghai Library”. Library Management 34 (1-2): 20-30.

Kostagiolas, P., A. Margiola and A. Avramidou. 2011. "A library management response model against the economic crisis: The case of public libraries in Greece". Library Review 60 (6): 486-500.

Leonard, E. and B. Clementson. 2012. "Business librarians and entrepreneurship: Innovation trends and characteristics". New Review of Information Networking 17 (1): $1-21$.

Müller, U. 2001. "Kritikmanagement als bestandteil einer marketing konzeption für bibliotheken”. Bibliothek Forschung und Praxis 25 (2): 214-225.

Munro, K., K. Stevenson, R. Stenson, W. Walker and C. Fisher. 2011. "Planning for the mobile library: a strategy for managing innovation and transformation at the University of Glasgow Library". Serials 24:26-31.

Oliveira, M. e Beatriz Valadares Cendón. 2005. Ciência da informação e biblioteconomia: novos conteúdos e espaços de atuação. Belo Horizonte: Editora ufmg.

Roy, B. 1993. "Decision science or decision-aid science?" European Journal of Operational Research 66 (2): 184-203.

Rowley, J. 2011. "Should your library have an innovation strategy?" Library Management 32 (4-5): 251-265.

Rzepczynski, M. 2013. "Expanding the boundaries of library work”. Public Libraries 52 (2) (march): 10-12.

Sampieri, R.H., C.F. Collado y P.B. Lucio. 1994. Metodología de la investigación. México: McGraw Hill.

Scherer, F.O. e M.S. Carlomagno. 2009. Gestão da inovação na prática. São Paulo: Atlas. Tidd, J., J. Bessant e K. Pavitt. 2008. Gestão da inovação. Porto Alegre: Bookman.

Para citar este texto:

Mauro Silveira, Murilo, William B. Vianna y Sandra Rolim Ensslin. 2018. "Gestão da inovação em bibliotecas: elementos fundamentais de revisão de literatura internacional”. Investigación Bibliotecológica: archivonomía, bibliotecología e información 32 (76): 29-44. http://dx.doi.org/10.22201/iibi.24488321xe.2018.76.57973 